\title{
Stimulation of the Calcitonin Gene-Related Peptide Enhancer by Mitogen-Activated Protein Kinases and Repression by an Antimigraine Drug in Trigeminal Ganglia Neurons
}

\author{
Paul L. Durham and Andrew F. Russo \\ Department of Physiology and Biophysics, University of Iowa, Iowa City, Iowa 52242
}

Calcitonin gene-related peptide (CGRP) is involved in the underlying pathophysiology of all vascular headaches, including migraines. Elevated levels of CGRP during migraine are restored to normal coincident with headache relief after treatment with the antimigraine drug sumatriptan. We have used primary cultures of trigeminal neurons under conditions simulating migraine pathology and therapy to study the mechanisms controlling the CGRP promoter. Using reporter genes in transient transfection assays, we demonstrate that an 18 bp enhancer containing a helix-loop-helix element is both necessary and sufficient for full promoter activity. NGF treatment and cotransfection with an upstream activator of the extracellular signal-regulated MAP kinases (MAPKs) activated the enhancer. Treatment with sumatriptan repressed NGF- and MAPK-stimulated CGRP promoter activity. Repression was also observed using a synthetic MAPK-responsive reporter gene. Sumatriptan regulation of CGRP gene expression did not couple to a $\mathrm{G}_{\mathrm{i}} / \mathrm{G}_{\mathrm{o}}$ pathway, but rather caused a prolonged increase in intracellular calcium. The importance of the prolonged calcium signal in repression of MAPK activity was demonstrated by using the ionophore ionomycin to mimic sumatriptan action. We propose that activation of MAPK pathways may increase CGRP gene expression during migraine, and that sumatriptan can diametrically oppose that activation via a prolonged elevation of intracellular calcium.

Key words: CGRP; trigeminal ganglia; MAPK; calcium; migraine; gene expression

\section{Introduction}

Calcitonin gene-related peptide (CGRP) is a multifunctional regulatory neuropeptide that is believed to play an important role in the underlying pathology of vascular headaches (Van Rossum et al., 1997). Serum levels of CGRP are elevated in patients during migraine and cluster headaches (Goadsby and Edvinsson 1993, 1994; Edvinsson and Goadsby, 1994; Fanciullacci et al., 1995). In the neurovascular model of migraine, trigeminal ganglia nerves are activated and release CGRP and other neuropeptides that mediate neurogenic inflammation within the meninges (Moskowitz, 1993; Buzzi et al., 1995; Williamson and Hargreaves, 2001). Interestingly, the wave of cortical-spreading depression during the migraine aura can activate the trigeminal ganglia (Bolay et al., 2002). Additional evidence for a role of trigeminal CGRP in migraine comes from clinical studies in which the serotonergic drug sumatriptan lowers CGRP levels in the jugular outflow, coincident with the relief of headache pain (Goadsby and Edvinsson, 1991). Because migraine can last for up to $3 \mathrm{~d}$, we reasoned that there might be a sustained increase in CGRP synthesis during neurogenic inflammation. Likewise, although the fast action of sumatriptan in patients is consistent with the inhi-

\footnotetext{
Received July 30, 2002; revised Nov. 4, 2002; accepted Nov. 12, 2002.

This work was supported by National Institutes of Health Grants HD 25969 and HL 14388, with tissue culture support provided by the Diabetes and Endocrinology Center (DK25295) and National Headache Foundation (P.L.D.)

Correspondence should be addressed to Andrew F. Russo, Department of Physiology and Biophysics, University of lowa, lowa City, IA 52242. E-mail: andrew-russo@uiowa.edu.

P. L. Durham's present address: Department of Biology, 225 Temple Hall, Southwest Missouri State University, Springfield, M0 65804. E-mail: pauldurham@smsu.edu.

Copyright $\odot 2003$ Society for Neuroscience $\quad$ 2270-6474/03/230807-09\$15.00/0
}

bition of secretion, we reasoned that there might also be a coordinate regulation at the level of transcription.

CGRP transcription is controlled by a cell-specific enhancer that contains a basic helix-loop-helix (bHLH) site and an adjacent octamer-binding motif (Peleg et al., 1990; Ball et al., 1992; Tverberg and Russo, 1993; Lanigan and Russo, 1997). This distal enhancer was identified using thyroid C-cell lines, which have neuronal-like properties (Russo et al., 1992, 1996). The promoter also contains a complex proximal element that is CAMP- and ras-responsive (de Bustros et al., 1986, 1992; Monia et al., 1995; Thiagalingam et al., 1996). It has been well established that CGRP levels are elevated by nerve growth factor (NGF) (Lindsay and Harmar, 1989; Verge et al., 1995; Patel et al., 2000; Shadiack et al., 2001; Supowit et al., 2001), and that NGF action appears to involve the $\mathrm{CAMP} / \mathrm{ras}$ element and an undefined region (Watson et al., 1995; Freeland et al., 2000). We have shown that the cellspecific CGRP enhancer is stimulated by depolarization and strongly activated ( $>10$-fold) by a constitutively active mitogenactivated protein kinase (MAPK) kinase (MEK1) in the CA77 thyroid C-cell line (Durham and Russo, 1998, 2000). Activation of serotonin type $1\left(5-\mathrm{HT}_{1}\right)$ receptors repressed basal and MEK1stimulated enhancer activity in the CA77 cells (Durham and Russo, 1998). However, although the CA77 cells are a good model, the question remained as to whether this regulation occurs in normal trigeminal neurons. To address this question, we have used primary cultures of rat trigeminal ganglia that we have shown previously to be responsive to sumatriptan and other $5-\mathrm{HT}_{1}$ agonists that inhibit CGRP secretion (Durham and Russo, 1999).

In the present study, we demonstrate that NGF and MAPK 
stimulation of CGRP promoter activity requires a single HLH site in the enhancer. These stimulatory effects were greatly repressed by sumatriptan treatment via a pertussis-toxin-insensitive pathway. Furthermore, the inhibitory effect of sumatriptan on promoter activity can be mimicked by a prolonged elevation in intracellular calcium. These results demonstrate that CGRP gene expression is stimulated by MAPKs and repressed by the antimigraine drug sumatriptan in trigeminal ganglia neurons.

\section{Materials and Methods}

Cell culture. Trigeminal ganglia cultures were based on our previous protocol (Durham et al., 1997). Ganglia isolated from $\sim 20-24$ 3- to 4 -d-old Sprague Dawley rats were washed in $10 \mathrm{ml}$ of cold plating medium [25 mM HEPES, pH 7.2-7.4, DMEM (high glucose)] and collected at $100 \times g$ for $2-3 \mathrm{~min}$. The ganglia were resuspended in $10 \mathrm{ml}$ of plating medium containing $10 \mathrm{mg} / \mathrm{ml}$ dispase II (Invitrogen, Gaithersburg, MD) and $1 \mathrm{U} / \mathrm{ml}$ RQ1 DNase (Promega, Madison, WI) and then split into two $15 \mathrm{ml}$ tubes $(5 \mathrm{ml} \mathrm{each})$ for $30 \mathrm{~min}$ at $37^{\circ} \mathrm{C}$. The cell suspension was collected by centrifugation at $100 \times g$ for $3 \mathrm{~min}$. The pellets were resuspended and further dissociated in $5 \mathrm{ml}$ of plating medium by vigorous trituration ( $\sim 15$ times) using a $5 \mathrm{ml}$ pipette. After allowing larger fragments to settle ( $\sim 1 \mathrm{~min})$, the suspensions from each tube were combined into a new $15 \mathrm{ml}$ tube. The remaining large fragments were pooled and triturated $\sim 15$ times in $5 \mathrm{ml}$ of plating medium. After the trituration, any remaining fragments were mechanically removed by gently swirling a Pasteur pipette in the suspension. The suspension was combined with the cells obtained after the first trituration step ( $15 \mathrm{ml}$ total) and centrifuged at $100 \times g$ for $4 \mathrm{~min}$. The cell pellet was resuspended in $6 \mathrm{ml} \mathrm{L15}$ (Leibovitz) medium containing $10 \%$ fetal bovine serum, $50 \mathrm{~mm}$ glucose, $250 \mu \mathrm{M}$ ascorbic acid, $8 \mu \mathrm{m}$ glutathione, $2 \mathrm{~mm}$ glutamine, and $10 \mathrm{ng} / \mathrm{ml}$ mouse 2.5 $\mathrm{S}$ NGF (Alomone Labs, Jerusalem, Israel) at $37^{\circ} \mathrm{C}$ at ambient $\mathrm{CO}_{2}$ levels. For transfection studies, the cells were plated on 12 well tissue culture plates (Becton Dickinson, Franklin Lakes, NJ) coated with poly-D-lysine (molecular weight, 30,000-70,000; Sigma, St. Louis, MO). Cultures were noticeably healthier on Becton Dickinson Falcon dishes compared with those of other manufacturers. Penicillin (100 U/ml), streptomycin (100 $\mu \mathrm{g} / \mathrm{ml})$, and amphotericin B $(2.5 \mu \mathrm{g} / \mathrm{ml}$; Sigma) were added to the L15 medium unless otherwise noted. For some studies, the cultures were enriched for neuronal cells by density-gradient centrifugation. After the dissociation step, the cell pellet was resuspended in $3 \mathrm{ml}$ of plating medium containing $1 \mathrm{mg} / \mathrm{ml}$ bovine serum albumin. The cells were carefully layered onto $6 \mathrm{ml}$ of plating medium containing $10 \mathrm{mg} / \mathrm{ml}$ bovine serum albumin in a $15 \mathrm{ml}$ conical tube, and then centrifuged at $100 \times g$ for 3 min. The cell pellet was resuspended in L15 medium and plated as described above. The culture medium was changed after $24 \mathrm{hr}$ and every other day thereafter. Bradykinin was purchased from Sigma; sumatriptan succinate (GlaxoSmithKline Pharmaceuticals, Harlow, UK) was obtained from the University of Iowa Pharmacy; and ionomycin, PD98059, U0124, and U0126 were supplied by Calbiochem-Novabiochem (La Jolla, CA). Pertussis toxin ( $100 \mathrm{ng} / \mathrm{ml}$; Invitrogen) treatments were for 20 $\mathrm{hr}$ before the experiment. In all studies, cells were treated with equivalent amounts of vehicle.

Immunohistochemistry. Transfected cultures were briefly rinsed in PBS and fixed in $100 \%$ methanol for $10 \mathrm{~min}$ at $-20^{\circ} \mathrm{C}$. The fixed cells were incubated for $30 \mathrm{~min}$ in PBS with $10 \%$ fetal bovine serum and then costained for $1 \mathrm{hr}$ with rabbit anti-rat CGRP polyclonal antibodies (1: 1000 dilution; gift from I. Dickerson, University of Miami, Miami, FL) and a mouse anti- $\beta$-galactosidase monoclonal antibody (1:1000 dilution; Promega). Secondary antibodies were rhodamine red X-conjugated donkey anti-rabbit IgG (1:100; The Jackson Laboratory, Bar Harbor, $\mathrm{ME})$ and FITC-conjugated donkey anti-mouse IgG (1:1000; The Jackson Laboratory).

Transfection of trigeminal cultures. All of the CGRP and herpes simplex thymidine kinase (TK) luciferase reporter plasmids and the cytomegalovirus (CMV) $\beta$-galactosidase reporter plasmid have been described previously (Tverberg and Russo, 1993; Lanigan and Russo, 1997). The 1250 bp CGRP promoter-luciferase reporter contains sequences from the $K p n I$ site $(-1250)$ to the Sau3A site $(+21)$ in exon 1. A BamHI linker
(CGGATCCG) was inserted at the PvuII site (-1038 bp) of the $1250 \mathrm{bp}$ CGRP luciferase gene (Tverberg and Russo, 1993). The 18 bp cell-specific enhancer [HLH and octamer-binding sites $(\mathrm{HO})]$ and $\mathrm{HO}+\mathrm{A}$ reporters contain four tandem repeats in the sense orientation. All plasmids were sequenced to confirm the insertions. The plasmids containing CMVMEK1 (S218/222E, $\Delta 32-51)$, the Elk-1 activation domain fused to the Gal4 DNA-binding domain, and the luciferase reporter with gall sites have been described previously (Durham and Russo, 1998).

Trigeminal ganglia cultures were transiently transfected with Lipofectamine 2000 (Invitrogen) according to the manufacturer's instructions. Approximately $3-5 \times 10^{4}$ cells (three to four ganglia) per well (12 well dish) were transfected $1 \mathrm{hr}$ after plating with 1-2 $\mu \mathrm{g}$ of CGRPluciferase reporter, $0.5 \mu \mathrm{g}$ of gall-luciferase reporter, $0.5 \mu \mathrm{g}$ of Gal4Elk-1, and/or $0.5 \mu \mathrm{g}$ of CMV-MEK1 plasmid DNAs. The amount of DNA was kept constant by the addition of the empty expression vector CMV-5 (Durham and Russo, 1998). The DNA and Lipofectamine 2000 reagent (ratio, $1 \mu \mathrm{g}: 3 \mu \mathrm{l}$ ) were incubated together for $20 \mathrm{~min}$ in L15 medium (without supplements). The DNA-Lipofectamine complex was then added to the trigeminal cultures maintained in the fully supplemented L15 medium (including serum, $10 \mathrm{ng} / \mathrm{ml} \mathrm{NGF}$ and +/- antibiotics, and an antifungal agent) and incubated for $\sim 24 \mathrm{hr}$ before assaying for reporter activity. Two hours before harvesting, $60 \mathrm{mM} \mathrm{KCl}, 10 \mu \mathrm{M}$ bradykinin, and $100 \mathrm{ng} / \mathrm{ml}$ NGF were added. In some experiments, the cells were preincubated for $30 \mathrm{~min}$ with sumatriptan, ionomycin, or the MAPK inhibitors PD98059, U0126, or U0124 before the addition of NGF. Cells transfected with MEK1 were incubated overnight in serumcontaining medium with sumatriptan or ionomycin and harvested $20 \mathrm{hr}$ later. Luciferase was measured using the Luciferase Assay System (Promega) and was reported as relative light units per $20 \mu \mathrm{g}$ of protein. Protein concentrations were determined by Bradford assays (Bio-Rad Laboratories, Hercules, CA). In all experiments, transfection efficiencies were normalized to $\mathrm{CMV}-\beta$-galactosidase activity that was measured using Galacto-Light reagents (Tropix, Bedford, MA). No difference in cell viability (trypan blue staining) was observed after transfection with the different plasmids or treatments. The normalized luciferase activities are reported as means with SEs per $20 \mu \mathrm{g}$ of protein. Each experimental condition was repeated in at least three independent experiments done in duplicate. Statistical analyses were done using Student's $t$ test.

Calcium measurements. Intracellular calcium levels in cultured trigeminal neurons were measured using a video microscope digital image analysis system (Photon Technology International Inc., South Brunswick, NJ) as described previously (Durham et al., 1997). Briefly, dissociated trigeminal ganglia cells grown on laminin-coated $25 \mathrm{~mm}$ glass coverslips were maintained in complete L15 serum-containing medium $24 \mathrm{hr}$ before the start of the calcium imaging procedure. Cells were incubated in L15 containing $0.2 \%$ bovine serum albumin and $1 \mu \mathrm{M}$ fura- $2 \mathrm{AM}$ (Molecular Probes, Eugene, OR) for $30 \mathrm{~min}$ at $37^{\circ} \mathrm{C}$ in ambient $\mathrm{CO}_{2}$. After washing the cells twice with HEPES-buffered saline, pH 7.4 (Durham and Russo, 1999), the cells were incubated for $30 \mathrm{~min}$ in L15 medium with insulin transferrin selenium (Sigma) (for sumatriptan studies) or L15 media with $10 \%$ serum (for ionomycin studies) before measurement using a Nikon (Tokyo, Japan) Diaphot microscope. Basal calcium levels were measured for a minimum of $120 \mathrm{sec}$ before treatment with sumatriptan or ionomycin. An equal volume of the appropriate medium containing sumatriptan or ionomycin (at two times the final concentration) was added directly to the cells, and the values were recorded every $20 \mathrm{sec}$, usually for $10-15 \mathrm{~min}$, on a heated stage at $37^{\circ} \mathrm{C}$. Calcium levels in individual trigeminal neurons as identified by morphological characteristics and response to depolarization with $60 \mathrm{~mm} \mathrm{KCl}$ were determined using a $50 \times 50$ pixel area in the center of each cell.

\section{Results \\ CGRP promoter activity in primary trigeminal ganglia neurons}

A 1250 bp rat CGRP promoter- $\beta$-galactosidase reporter plasmid was used to detect transiently transfected neurons in primary trigeminal ganglia cultures (Fig. 1). This promoter includes an 18 bp cell-specific enhancer that is necessary and sufficient for cell- 


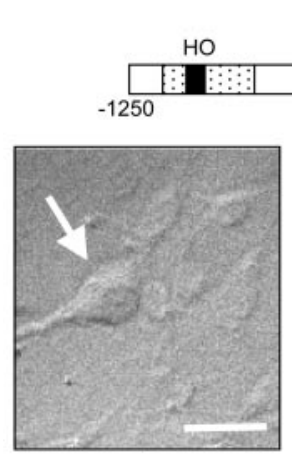

CGRP

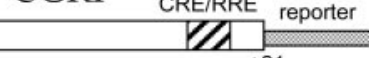

$+21$
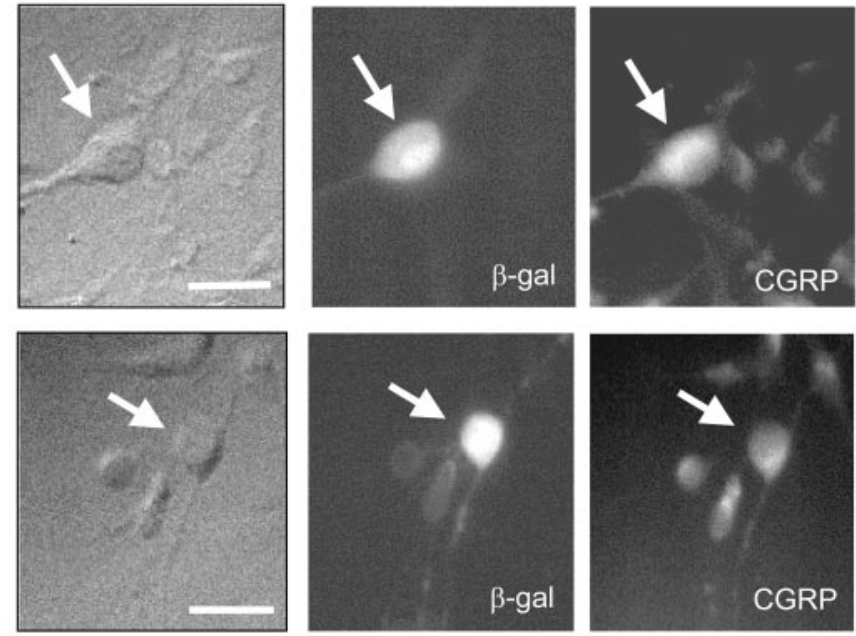

Figure 1. Expression of a CGRP promoter- $\beta$-galactosidase reporter gene in trigeminal ganglia cultures. A schematic of the $1250 \mathrm{bp}$ rat CGRP promoter fragment showing the proximal cAMP-response element (CRE) and ras-response element (RRE) responsive regions (striped) and the distal enhancer that contains both cell-specific 18 bp HO (solid) and non-cell-specific (spotted) elements is shown at the top. Trigeminal cultures were transfected with the $1250 \mathrm{bp}$ CGRP- $\beta$-galactosidase reporter gene and then analyzed $24 \mathrm{hr}$ after transfection. The cells were visualized by Nomarski optics (left) and double stained for $\beta$-galactosidase ( $\beta$-gal; middle) and CGRP (right) immunoreactivity. The cell body of a neuronal cell is identified by an arrow in each set of photographs. Scale bars, $50 \mu \mathrm{m}$.

specific expression in rat and human thyroid C-cell lines (Tverberg and Russo, 1993; Lanigan and Russo, 1997). The expression of $\beta$-galactosidase and CGRP in the transfected cultures was determined by counting the number of positive cells from several fields from each of the dishes with a $10 \times$ objective (total magnification, $100 \times)$. The $\beta$-galactosidase reporter was usually detected in $5-10 \%$ ( 25 of 279 ) of cells present in the day 1 cultures (Fig. 1). Of the cells counted from several dishes, most ( $>90 \% ; 23$ of 25) of the $\beta$-galactosidase-positive cells were identified as neuronal based on morphology (round cell body of 20-50 $\mu \mathrm{m}$ ) and colocalization with CGRP. Identification of neuronal cells based on the presence of neurites was not reliable, because many of the CGRP-containing neurons did not exhibit visible processes until day 2 in culture. A serendipitous consequence of the transfection protocol was the enrichment of neuronal cells $(>90 \% ; 136$ of $148 ; n=6$ dishes). This enrichment is likely a result of toxicity to replicating cells when antibiotics are present in the media during transfection using Lipofectamine 2000. The percentage of neuronal cells in the absence of antibiotics was much lower $(\sim 40 \% ; 39$ of $98 ; n=3$ dishes). These data demonstrate that the $1250 \mathrm{bp}$ CGRP promoter is sufficient to direct reporter gene expression in trigeminal neurons.

To test whether the cell-specific enhancer that had been identified in neuronal-like cell lines was active in trigeminal neurons, we used a series of CGRP promoter-luciferase reporters. CGRP promoter activity was greatly diminished by insertion of an $8 \mathrm{bp}$ BamHI linker into the HLH motif of the HO enhancer (Fig. 2A). A reporter with the TK promoter and the CGRP $5^{\prime}$ region from -1120 to -920 , which contains the HO element and flanking non-cell-specific elements, had threefold to fourfold greater activity than the TK promoter alone (Fig. 2 B). A similar increase over TK was observed with a reporter containing four copies of

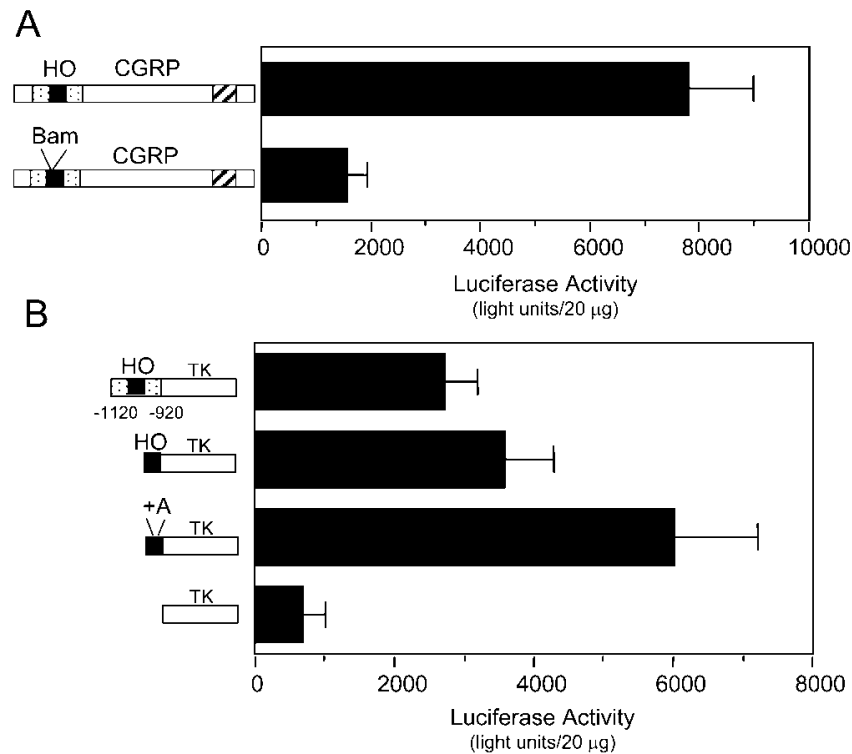

Figure 2. Mapping of basal CGRP enhancer activity in trigeminal neurons. $A$, The 1250 bp CGRP promoter-luciferase reporter gene was transfected into primary trigeminal cultures, and luciferase activity was measured after $24 \mathrm{hr}$. Insertion of a BamH linker into the $\mathrm{HLH}$ motif in the HO enhancer greatly reduced reporter activity. $B$, Reporter genes containing the minimal TK promoter with either a single $200 \mathrm{bp}$ region containing the $\mathrm{HO}$ enhancer or a multimer of the 18 bp HO enhancer yielded luciferase activity greater than that of the TK promoter alone. Insertion of a single adenosine between the HLH and octamer motif $(+A)$ did not reduce the activity of the multimerized $\mathrm{HO}$ element. The normalized means with SEs from at least three independent experiments are shown.

the HO element. These results are in agreement with previous cell-line studies. However, a mutation in the octamer-binding motif that had been shown previously to block enhancer activity almost completely in thyroid C-cell lines (Durham et al., 1997) did not inhibit enhancer activity, but rather, slightly stimulated activity in the trigeminal cultures (Fig. $2 B$ ). This mutation is an insertion of an adenosine adjacent to the HLH motif, which generates a consensus octamer site. These data indicate that although the role of the octamer site is not clear, the HLH site of the HO enhancer is required for basal enhancer activity in trigeminal neurons.

\section{Stimulation of CGRP promoter activity by depolarization, proinflammatory agents, and MAPKs}

The effect of trigeminal neuron activation on CGRP promoter activity was investigated using several paradigms. Treatment with $\mathrm{KCl}$ to mimic neuronal depolarization caused a more than twofold increase in promoter activity (Fig. 3A). A similar effect on promoter activity was observed after treatment with bradykinin, a pronociceptive agent that causes inflammation in peripheral tissues (Davis and Dostrovsky, 1988; Kai, 1993). CGRP promoter activity was markedly stimulated (almost fivefold) by the proinflammatory agent NGF that is involved in chronic pain conditions and elevated during headaches (Bennet, 2001; Sarchielli et al., 2001). These results demonstrate that CGRP promoter activity is stimulated in cultured trigeminal neurons by factors known to activate sensory neurons and promote inflammation.

The mechanism of NGF activation of the CGRP enhancer was then determined. We focused on the MAPK pathway, because the NGF receptor can activate MAPKs in other systems (Ahn et al., 1992; Pang et al., 1995) and we have shown that the enhancer is MAPK-responsive in the CA77 cells (Durham et al., 1998, 2000). 

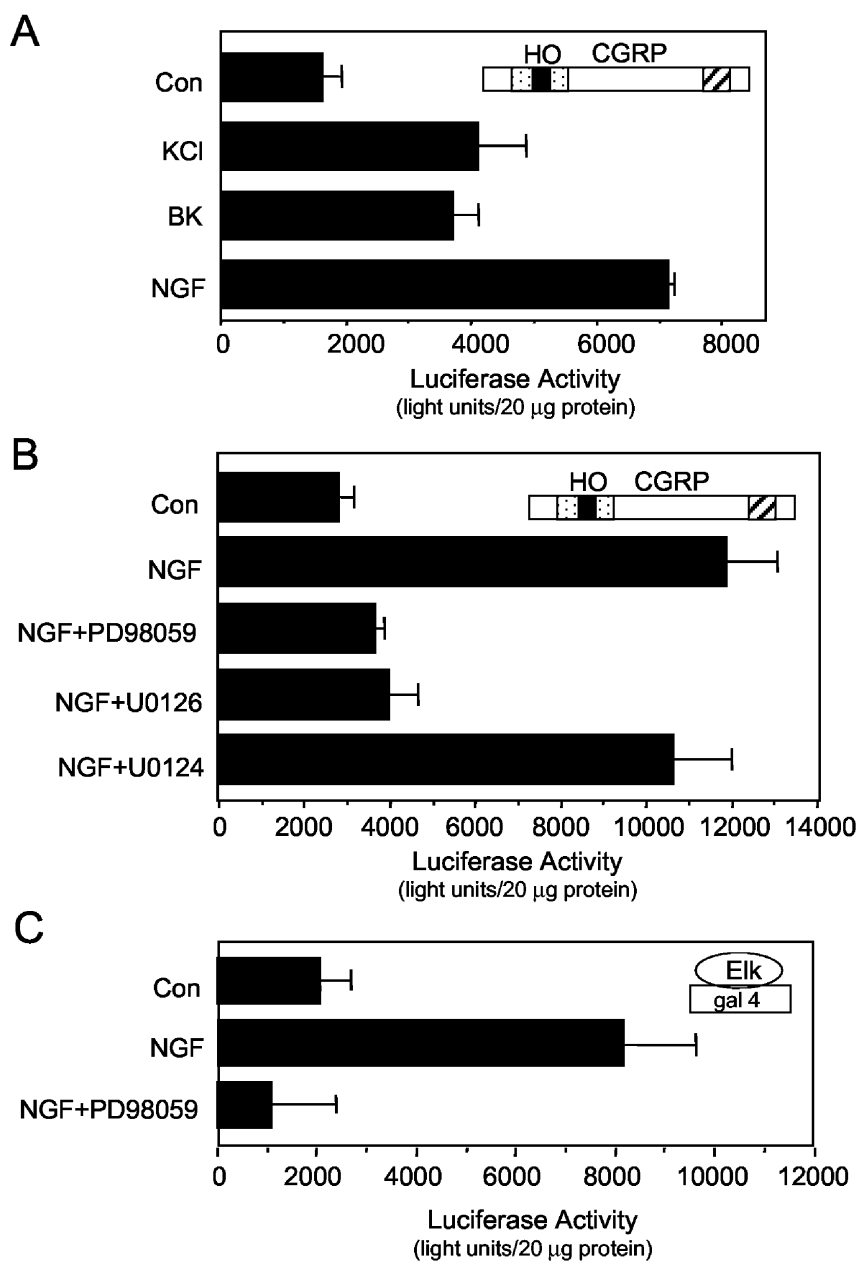

Figure 3. NGF stimulation of CGRP promoter activity via ERK MAPK. A, Trigeminal cultures transfected with the $1250 \mathrm{bp}$ CGRP reporter were untreated [controls (Con)] or treated with 60 $\mathrm{mm} \mathrm{KCl}, 10 \mu \mathrm{m}$ bradykinin (BK), or $100 \mathrm{ng} / \mathrm{ml} \mathrm{NGF}$ for 2 hr before harvest. $B$, Trigeminal cultures transfected with the $1250 \mathrm{bp}$ (GRP reporter were treated with NGF in the absence or presence of the MEK inhibitors PD98059 (10 $\mu \mathrm{m})$, U0126 $(1 \mu \mathrm{M})$, or the inactive control U0124 (10 $\mu \mathrm{M})$ for $2 \mathrm{hr}$ before harvest. C, The effect of $100 \mathrm{ng} / \mathrm{ml}$ NGF and PD98059 on Elk-1 reporter gene activity is shown. The normalized means with SEs from at least four independent experiments for each panel are shown.

CGRP promoter activity was measured from cultures cotreated with NGF and two different inhibitors (PD98059 and U0126) of MEK1 and MEK 2, which selectively activate only the extracellular signal-regulated MAPKs ERK1 and ERK2. Treatment with the MEK1/2 inhibitors greatly diminished the stimulatory effect of NGF (Fig. 3B). As a control, no appreciable change in promoter activity was observed with the inactive analog U0124. NGF activation of MAPK pathways was confirmed by an independent test using a synthetic reporter gene that is activated by the known MAPK-responsive transcription factor Elk-1. NGF stimulated Elk-1 reporter activity approximately fourfold (Fig. 3C). As a control, PD98059 cotreatment blocked the NGF stimulation. These data establish that NGF activation of an ERK MAPK pathway is required for the activation of the CGRP promoter in cultured trigeminal neurons.

Because NGF can activate multiple signaling pathways, we then tested whether ERK MAPK activation is sufficient to activate the CGRP promoter. Trigeminal cultures were cotransfected with the CGRP promoter reporter plasmid and a plasmid encoding a constitutively active MEK1. Coexpression of activated
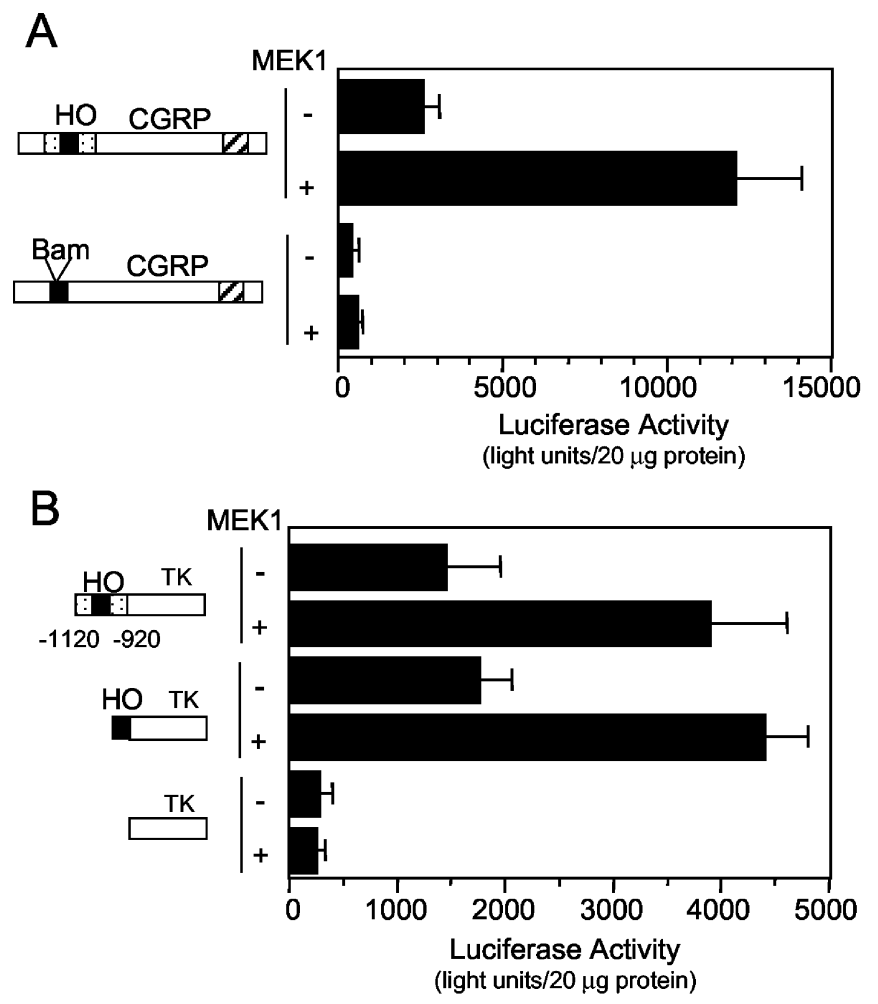

Figure 4. MEK1 stimulation of the CGRP HO enhancer. $A$, Trigeminal cultures were cotransfected with the $1250 \mathrm{bp}$ CGRP-luciferase reporter or the $1250 \mathrm{bp}$ reporter with the HLH mutation (Bam insertion) with or without a CMV-MEK1 expression vector. The activity of the $1250 \mathrm{bp}$ promoter, but not the mutant promoter, was increased by MEK1. B, Activation of the CGRP HO enhancer within the $200 \mathrm{bp}$ region or as a multimerized $18 \mathrm{bp}$ element linked to the TK promoter by cotransfected MEK1. The normalized means with SEs from at least three independent experiments are shown.

MEK1 increased CGRP promoter activity almost fivefold over control (Fig. 4A).

To identify the target sequence of the MEK1-activated signaling pathway, we tested the effect of MEK1 on the 1250 bp CGRP promoter fragment containing the mutated enhancer (Fig. 4A). Mutation of the HLH site of the HO enhancer almost eliminated the stimulatory effect of MEK1. In addition to being required for MAPK responsiveness, the $\mathrm{HO}$ enhancer was also sufficient to mediate the effect of MEK1. MEK1 caused a severalfold stimulation of two reporters containing either the -1120 to $-960 \mathrm{bp}$ enhancer fragment or a multimer of the $18 \mathrm{bp} \mathrm{HO}$ enhancer, whereas no effect was seen on the control TK promoter activity (Fig. 4B). These data demonstrate that MEK1 stimulation of the CGRP promoter is mediated via the HLH site in the HO enhancer.

\section{Repression of stimulated CGRP promoter activity}

by sumatriptan

Having shown that the $\mathrm{HO}$ enhancer is stimulated by MAPKs, we then determined whether treatment with the antimigraine drug sumatriptan could repress this activation. As seen in Figure $5 A$, increasing concentrations of sumatriptan correlated with greater repression of the MAPK-activated enhancer. At $0.1 \mu \mathrm{M}$, CGRP activity was reduced to $\sim 70 \%$ of the MEK1-stimulated activity. Repression to $27 \%$ of the MEK1 value was observed at $10 \mu \mathrm{M}$ sumatriptan. These results are in agreement with the dose dependence of sumatriptan repression of stimulated CGRP release from cultured trigeminal neurons (Durham and Russo, 1999). 
A

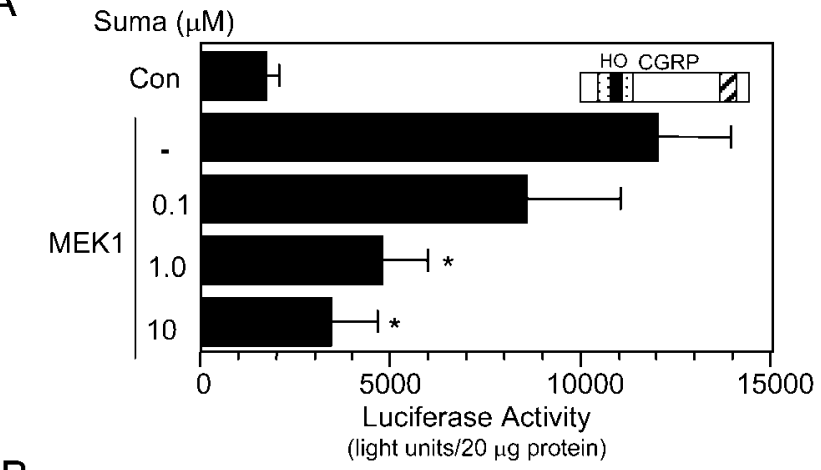

B

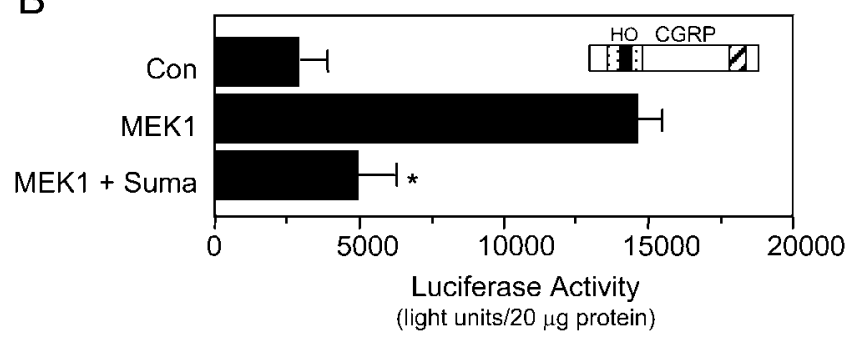

Figure 5. Sumatriptan (Suma) repression of MEK1 stimulation of the CGRP reporter genes. $A$, Trigeminal cultures transfected with only the $1250 \mathrm{bp}$ CGRP-luciferase reporter gene or cotransfected with CMV-MEK1 expression vector were either untreated ( - ) or treated overnight with the indicated amounts of sumatriptan, which lowered reporter activity. $B$, Trigeminal cultures were treated as in $A$, except for incubation overnight in antibiotic- and antifungicidefree media. The cultures were treated with $10 \mu$ m sumatriptan. The normalized means with SES from at least three independent experiments are shown. ${ }^{*} p<0.01$ compared with MEK1stimulated values. Con, Controls.

Typically, cultures were transfected and incubated overnight in media containing antibiotics, which as noted previously, resulted in an enrichment for neurons. As a control, we also tested promoter activity in the absence of antibiotics and amphotericin after enrichment for neurons by density centrifugation. MEK1 stimulation and repression by sumatriptan of CGRP promoter activity was very similar for these enriched cultures in the absence of antibiotics and antifungal agents (Fig. 5B).

Consistent with the repression of MEK1 activation, $10 \mu \mathrm{M}$ sumatriptan also repressed NGF stimulation of the CGRP promoter (Fig. 6A). To determine whether sumatriptan caused a general repression of MAPK responsiveness, the effect on Elk-1 transactivation activity was investigated. Sumatriptan treatment markedly decreased NGF stimulation of the MAPK-responsive Elk-1 transactivation domain (Fig. 6B).

\section{Sumatriptan repression is pertussis-toxin-insensitive}

To begin to understand the mechanism by which sumatriptan represses MAPK-stimulated CGRP gene expression, transfected trigeminal cultures were treated with pertussis toxin before sumatriptan treatment. Sumatriptan has a high affinity for the $5-\mathrm{HT}_{1}$ class of receptors that have been reported to inhibit adenylate cyclase via the pertussis-toxin-sensitive $G_{i} / G_{0}$ proteins (Boess and Martin, 1994). However, pertussis toxin did not block sumatriptan-mediated repression of the CGRP promoter in cultured trigeminal neurons (Fig. 7). This finding is in agreement with our previous observations that trigeminal neuron $5-\mathrm{HT}_{1}$ receptors are apparently not pertussis-toxin-sensitive and do not decrease intracellular cAMP levels (Durham and Russo, 1999).
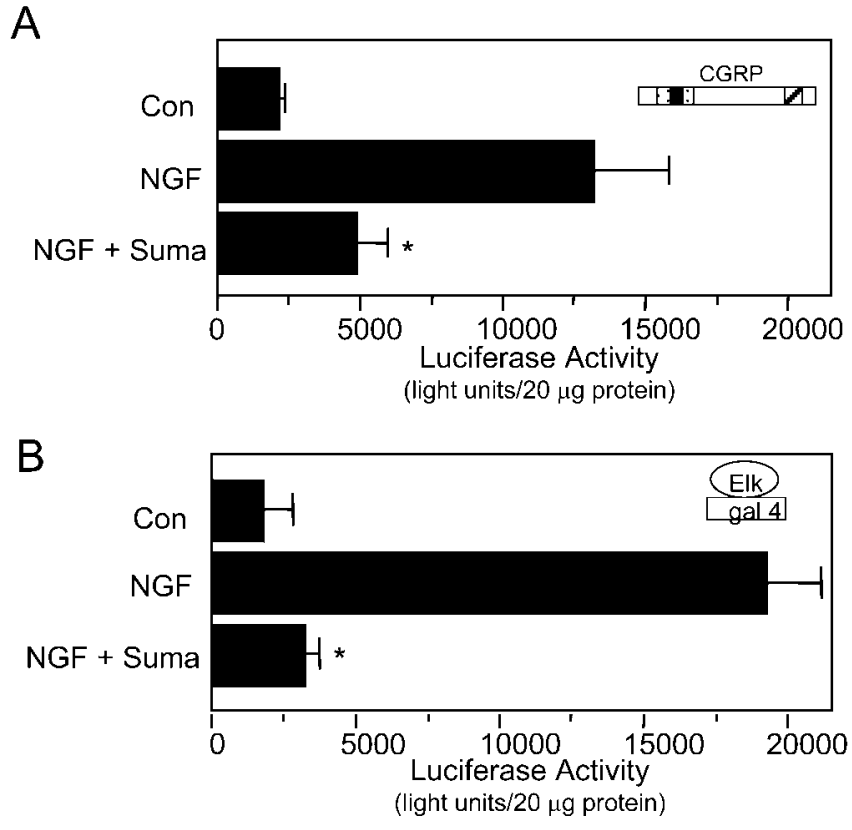

Figure 6. Sumatriptan (Suma) repression of NGF stimulation of the CGRP and Elk-1 reporter genes. $A$, Trigeminal cultures transfected with the $1250 \mathrm{bp} \mathrm{CGRP-luciferase} \mathrm{reporter} \mathrm{gene} \mathrm{were}$ either untreated, treated with NGF for $2 \mathrm{hr}$, or pretreated with $10 \mu \mathrm{m}$ sumatriptan for $30 \mathrm{~min}$ before NGF addition for $2 \mathrm{hr}$ before harvest. $B$, Elk-1 reporter activity in cultures treated with NGF and sumatriptan as in $A$ was repressed by sumatriptan treatment. The normalized means with SEs from at least three independent experiments are shown. ${ }^{*} p<0.01$ compared with stimulated values. Con, Controls.

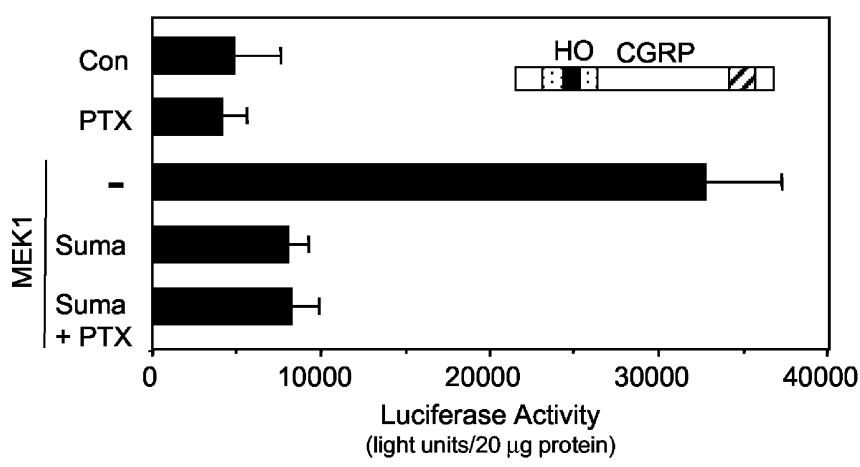

Figure 7. Pertussis toxin treatment does not block the effect of sumatriptan (Suma). Trigeminal cultures transfected with the CGRP reporter gene and MEK1 expression vector were treated with sumatriptan overnight alone or with $100 \mathrm{ng} / \mathrm{ml}$ pertussis toxin (PTX). The luciferase activities are the normalized means with SEs from at least three independent experiments. Con, Controls.

Sumatriptan causes prolonged elevation of intracellular calcium

We have previously observed a prolonged increase in calcium after sumatriptan treatment of trigeminal neurons (Durham et al., 1997; Durham and Russo, 1999). Because the culture conditions used in this study differ from previous investigations, we first confirmed that sumatriptan caused a steady and prolonged increase in intracellular calcium levels (Fig. 8A). As seen previously, there was a lag and considerable heterogeneity in the responses. Approximately one-half of the neurons responded with increased calcium levels that reached a plateau of $\sim 200 \mathrm{nM}$ after 5 min (Fig. $8 \mathrm{~B}$ ). The percentage of cells shown to be sumatriptanresponsive correlates with our observation that $5-\mathrm{HT}_{1 \mathrm{~B}}, 5-\mathrm{HT}_{1 \mathrm{D}}$, and $5-\mathrm{HT}_{1 \mathrm{~F}}$ receptors are expressed at varying levels in approxi- 


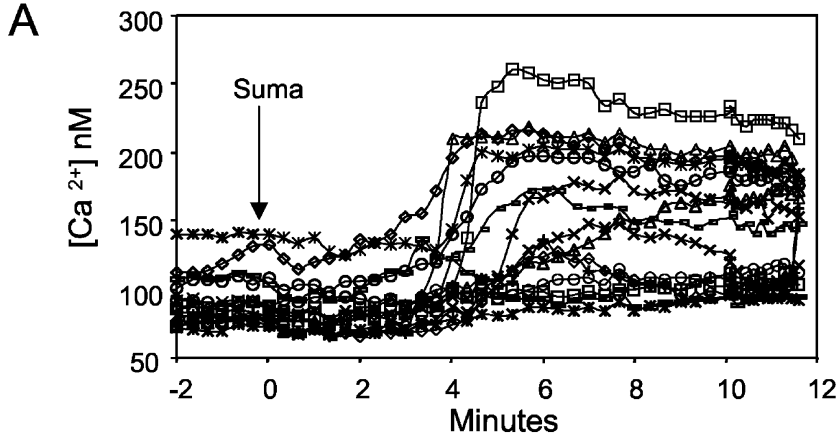

B

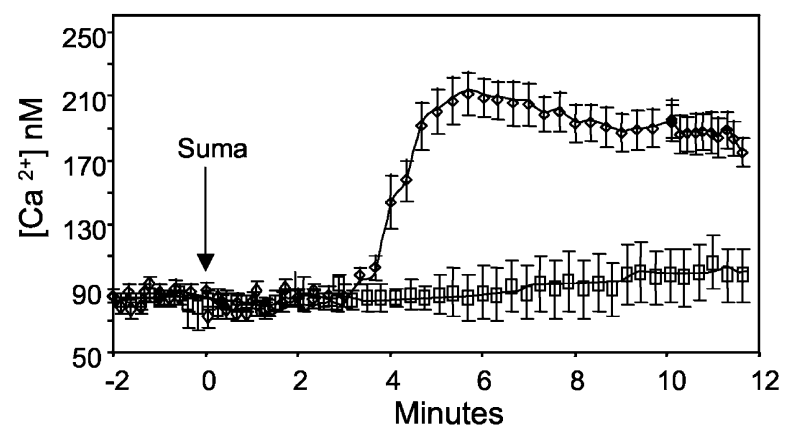

C

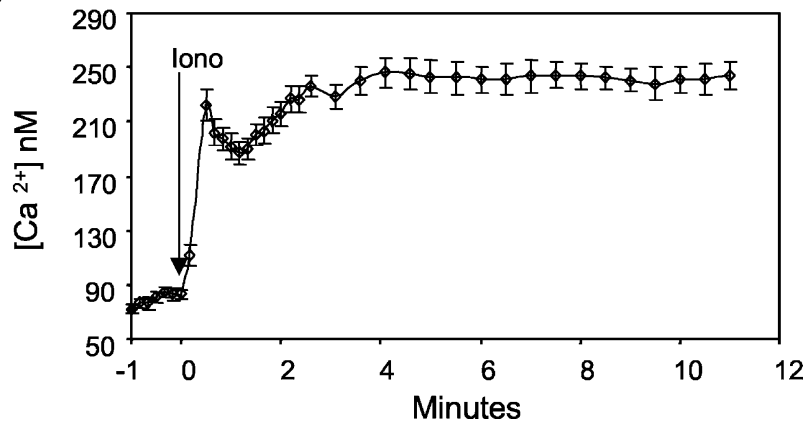

Figure 8. Sumatriptan (Suma) and ionomycin (Iono) cause prolonged elevation of intracellular calcium in trigeminal neurons. Calcium concentrations $\left[\mathrm{Ca}^{2+}\right]$ from day 2 cultures were measured using fura- 2 and a microscopic digital imaging system. $A$, The calcium concentrations of individual neurons in response to $10 \mu \mathrm{m}$ sumatriptan are shown ( $n=14$ cells from 2 independent experiments). $B$, The means and SEs in calcium levels are shown for sumatriptanresponsive cells (designated as $>150 \mathrm{~nm}$ calcium response; $n=8$ ) and the nonresponsive neurons $(n=6)$ from $A$. C, All neurons responded to $1 \mu$ m ionomycin. The means and SEs are shown ( $n=34$ cells from 3 independent experiments).

mately one-half of the neurons in our cultures (data not shown). The heterogeneous expression of $5-\mathrm{HT}_{1}$ receptors is in agreement with the results of Hou et al. (2001) from studies on human trigeminal ganglia. The sumatriptan-mediated elevation in intracellular calcium could be maintained for as long as $2 \mathrm{hr}$, the longest time point examined (data not shown). Although the duration of the calcium increase was similar to that in our previous reports, the amplitude of the increase was less. The difference may be attributable to the effect of transfection on the cells and/or the shorter culture time ( 2 vs $4 \mathrm{~d}$ ). For comparison, treatment of the cultures with the calcium ionophore ionomycin $(1 \mu \mathrm{M})$ caused an elevation of calcium in all neuronal cells that had a faster onset, but was otherwise similar in amplitude and duration to that observed using sumatriptan (Fig. 8C).

Elevated calcium levels are sufficient to repress the CGRP promoter

To test directly whether the sustained calcium elevation is responsible for sumatriptan repression of the CGRP pro-
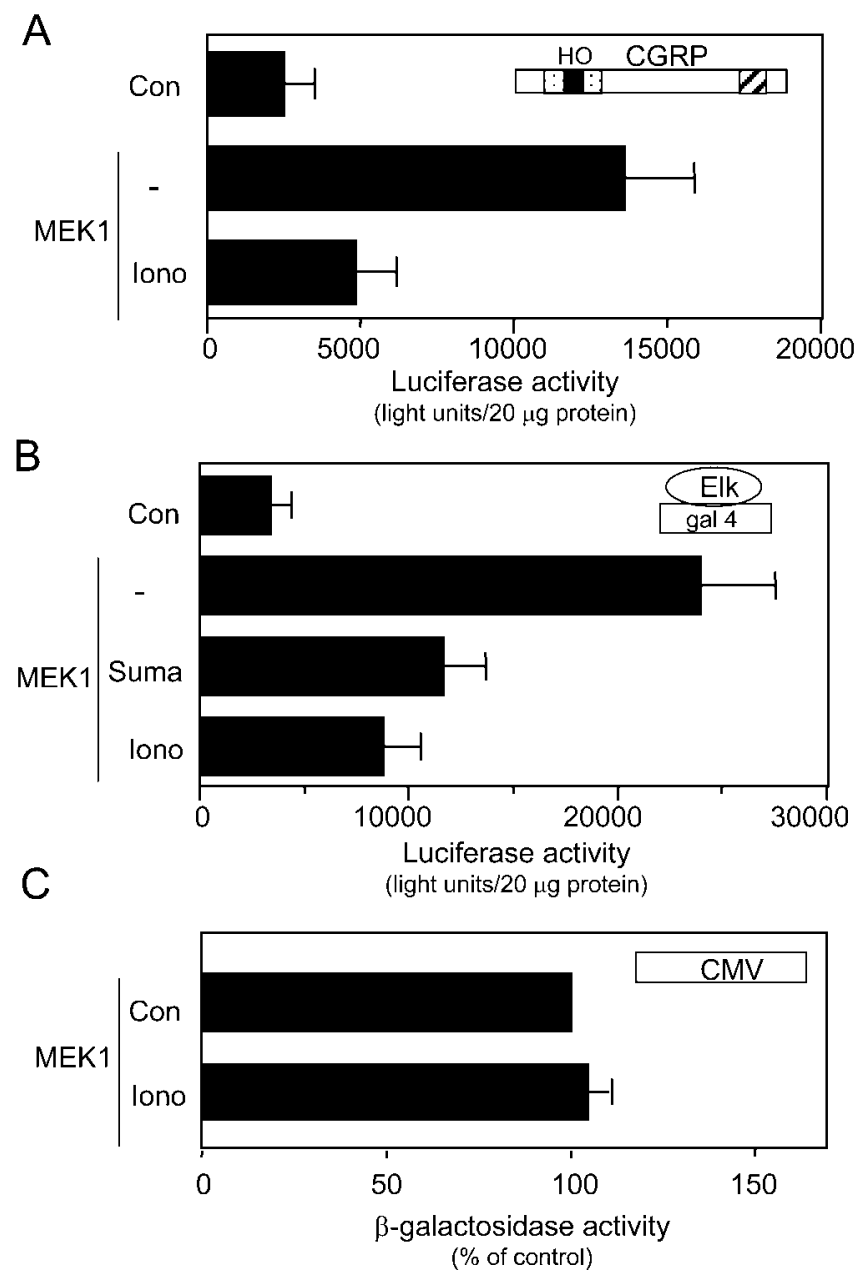

Figure 9. Ionomycin treatment represses MEK1 stimulation of CGRP and Elk-1 reporter genes. A, Cells were cotransfected with the $1250 \mathrm{bp}$ CGRP reporter and CMV-MEK1 expression vector and then treated overnight with $1 \mu \mathrm{m}$ ionomycin (Iono). B, Repression of Elk-1 reporter gene activity by treatment overnight with $10 \mu \mathrm{m}$ sumatriptan and $1 \mu \mathrm{m}$ ionomycin. The luciferase activities are the normalized means with SEs from at least three independent experiments. C, As a control, the CMV- $\beta$-galactosidase reporter activities were not affected by the overnight ionomycin treatment. Activities from the ionomycin-treated cultures were normalized to the control dishes that were set at $100 \%$ for each experiment $(n=6)$. The mean and SE is shown. Con, Controls.

moter, trigeminal cultures were treated with ionomycin to mimic the calcium increase caused by sumatriptan. Overnight, ionomycin treatment repressed MEK1-stimulated CGRP promoter activity to $\sim 35 \%$ of the activity of MEK1 alone (Fig. 9A). The degree of repression observed with ionomycin was similar to that seen with sumatriptan (Fig. 5A). It was essential that the treatment was done in the presence of serum to avoid cell toxicity that was otherwise seen within minutes of ionomycin application.

As with sumatriptan, we tested whether the ionomycin treatment repressed the synthetic MAPK-responsive reporter gene. Treatment with ionomycin markedly decreased MEK1 stimulation of the Elk reporter gene, similar to that seen with sumatriptan treatment (Fig. 9B). As a control, ionomycin treatment did not affect the cotransfected $\mathrm{CMV}-\beta$-galactosidase reporter gene (Fig. 9 C). These data demonstrate that a prolonged increase in intracellular calcium is sufficient to repress MAPKresponsive genes. 


\section{Discussion}

In this study, we have shown that CGRP gene expression in trigeminal ganglia neurons is upregulated by proinflammatory mediators and repressed by an antimigraine drug. Migraine is a painful neurological disorder that afflicts $16 \%$ of the general population (Stewart et al., 1994; Ferrari, 1998). Although the specific cause remains unknown, current theories suggest that the initiation of migraine involves a primary CNS dysfunction with subsequent activation of the trigeminovascular system (Burnstein, 2001; Buzzi, 2001; Bolay et al., 2002). Activation of trigeminal neurons is known to elevate CGRP levels during migraine (Edvinsson and Goadsby, 1994; Williamson and Hargreaves, 2001). The ability of acute antimigraine drugs such as sumatriptan to return serum CGRP levels to normal coincident with alleviation of pain is suggestive that CGRP is involved in the underlying pathology of migraine (Goadsby and Edvinsson, 1991). More recently, evidence in support of a causative role for CGRP in migraine was demonstrated by an in vivo study in which the administration of CGRP was shown to cause headache and migraine in migraineurs (Lassen et al., 2001).

Because an untreated migraine can persist for up to $72 \mathrm{hr}$, it seems likely that increased CGRP synthesis would be required to maintain elevated levels. Our results indicate that proinflammatory agents, such as NGF, can stimulate CGRP promoter activity in trigeminal neurons. These observations are consistent with the proposal that CGRP release during migraine results in the production and/or release of agents that escalate and sustain the inflammatory response (Williamson and Hargreaves, 2001). It is interesting to note that both NGF and CGRP levels appear to be elevated in the CSF of patients with chronic daily headache (Sarchielli et al., 2001). Our findings are in overall agreement with previous studies showing that NGF stimulates CGRP expression (Lindsay and Harmar, 1989; Verge et al., 1995; Watson et al., 1995; Freeland et al., 2000; Patel et al., 2000; Shadiack et al., 2001; Supowit et al., 2001). We have extended those studies by showing that the CGRP HO enhancer is an NGF-responsive target in trigeminal neurons. Previous results from dorsal root ganglia and PC12 cells have demonstrated a requirement for the downstream cAMP/ras-responsive element for activation by NGF (Watson et al., 1995; Freeland et al., 2000), and our findings do not rule out a role for this element in NGF responsiveness. Instead, we suggest that it may act with the $\mathrm{HO}$ element and be important for proinflammatory signals mediated by other MAPKs, because in our system the cAMP/ras element could be activated by a constitutively active MEK kinase 1 (amino acids $380-672$ ) (data not shown). In this regard, there is a recent report that both the bHLH protein upstream stimulatory factor-1 (USF-1)/USF-2 and cAMP-responsive element binding protein contribute to $\mathrm{KCl}$-induced activation of the BDNF promoter (Tabuchi et al., 2002). Together, these results suggest that at least two elements are activated by NGF and MAPK pathways, which provide a positive feedback mechanism for maintaining a prolonged elevation of CGRP during migraine (Fig. 10).

Within the HO enhancer, the target of the ERK MAPK pathway appears to be a bHLH transcription factor. The bHLH factors are well documented as key regulators of neuronal phenotype (Ma et al., 1998; Cau et al., 2002). Within the past year, bHLH factors have also been shown to be directly regulated by MAPK pathways (Bain et al., 2001; Galibert et al., 2001). For example, USF-1 is phosphorylated and activated by proinflammatory agents via MAPK p38 (Galibert et al., 2001). A heterodimer of USF-1/USF-2 can bind the HO HLH site in vitro (Lanigan and

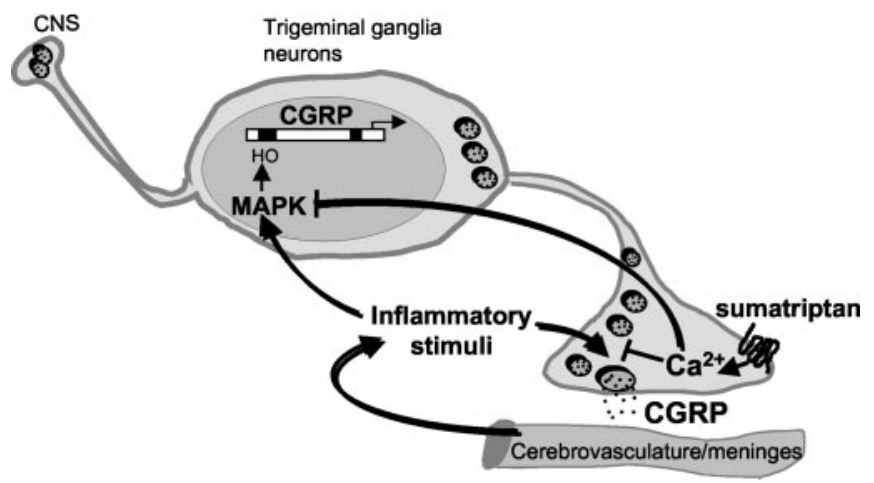

Figure 10. Model of CGRP regulation in trigeminal ganglia neurons under conditions simulating migraine pathology and therapy. Activation of trigeminal nerves leads to the initial release of CGRP and other neuropeptides that release proinflammatory mediators. These stimuli further augment CGRP synthesis and secretion via MAPKs. The antimigraine drug sumatriptan can cause a prolonged elevation of intracellular calcium that blocks the MAPK activation of CGRP synthesis and release.

Russo, 1997). Whether USF-1 binds the HO site in trigeminal neurons or if the p38 pathway can stimulate the enhancer remains to be tested. The bHLH requirement for basal and MAPKstimulated enhancer activity is in agreement with our results from the neuronal-like CA77 thyroid C-cell line (Durham et al., 1997). However, one difference appears to be the requirement for an adjacent octamer-binding protein. A mutation in the octamer-binding site $(18 \mathrm{bp}+\mathrm{A})$ that virtually eliminated activity in CA77 cells (Durham et al., 1997), had no deleterious effect on enhancer activity in the trigeminal cultures. Thus although the HLH site is clearly important, the octamer motif is either recognized by a different protein or is not required in trigeminal neurons.

A key finding of this study is that sumatriptan can repress the activation of the CGRP promoter by increasing intracellular calcium levels (Fig. 10). We reported previously that activation of $5-\mathrm{HT}_{1}$ receptors by sumatriptan causes a markedly prolonged increase in intracellular calcium in trigeminal neurons (Durham et al., 1997; Durham and Russo, 1999). We have now demonstrated a causal role for that prolonged calcium elevation. Using a calcium ionophore, we were able to mimic the amplitude and duration of the calcium increase observed after sumatriptan treatment. The prolonged calcium elevation was sufficient to repress MAPK-stimulated CGRP promoter activity. Interestingly, there is evidence that bHLH binding can be inhibited by elevated calcium in response to receptor activation or ionomycin (Corneliussen et al., 1994; Onions et al., 2000). Thus, calcium-induced repression of CGRP gene expression may involve changes in the phosphorylation state of bHLH transcription factors that act at the $\mathrm{HO}$ enhancer.

The regulation of CGRP has important implications for migraine pathology and therapy. In addition to the transcriptional regulation in this study, we have shown previously that depolarization and proinflammatory agents cause a marked increase in CGRP release that can be repressed by sumatriptan (Durham and Russo, 1999). Thus, it appears that trigeminal neurons are able to regulate coordinately both the synthesis and secretion of CGRP in response to stimulatory and inhibitory signals. The release of CGRP was repressed within $1 \mathrm{hr}$ after sumatriptan treatment, which is in agreement with the reported time of sumatriptan action in patients. The concentration of sumatriptan that is required to repress either CGRP secretion or transcription is 5-50 times greater than the estimated sumatriptan levels in the plasma 
of patients $(0.2 \mu \mathrm{M})$ (Fowler et al., 1991). It is interesting that Eltorp et al. (2000) also found that high sumatriptan doses $(10-50 \mu \mathrm{M})$ were required to repress CGRP release in a study using an in situ preparation of dura mater and trigeminal nerves. Possible explanations for these relatively high dose requirements may be that receptor levels or localization are different in culture and/or the stimulation paradigm is stronger in vitro. In either case, the inhibition of transcription is unlikely to affect CGRP levels in perivascular fibers in the clinical time frame of sumatriptan action. Instead, we speculate that a transcriptional mechanism could help maintain lower CGRP levels in the face of stimulatory signals. The finding that MAPKs stimulate the CGRP enhancer in primary trigeminal neurons may be particularly relevant to migraine pathology, because many proinflammatory agents implicated in migraines are known to activate MAPK pathways (Durham and Russo, 2002). We suggest that targeted and selective delivery of inhibitors of MAPK pathways to trigeminal neurons may provide a novel approach for dampening CGRP levels.

\section{References}

Ahn NG, Robbins DJ, Haycock JW, Seger R, Cobb MH, Krebs EG (1992) Identification of an activator of the microtubule-associated protein $2 \mathrm{ki}$ nases ERK1 and ERK2 in PC12 cells stimulated with nerve growth factor or bradykinin. J Neurochem 59:147-156.

Bain G, Cravatt CB, Loomans C, Alberola-Ila J, Hedrick SM, Murre C (2001) Regulation of the helix-loop-helix proteins, E2A and Id3, by the Ras-ERK MAPK cascade. Nat Immunol 2:165-171.

Ball DW, Compton D, Nelkin BD, Baylin SB, deBustros A (1992) Human calcitonin gene regulation by helix-loop-helix recognition sequences. $\mathrm{Nu}$ cleic Acids Res 20:117-1230.

Bennet DL (2001) Neurotrophic factors: important regulators of nociceptive function. Neuroscientist 7:13-17.

Boess FG, Martin IL (1994) Molecular biology of 5-HT receptors. Neuropharmacology 33:275-317.

Bolay H, Reuter U, Dunn AK, Huang Z, Boas DA, Moskowitz MA (2002) Intrinsic brain activity triggers trigeminal meningeal afferents in a migraine model. Nat Med 8:136-142.

Burnstein R (2001) Deconstructing migraine headache into peripheral and central sensitization. Pain 89:107-110.

Buzzi MG (2001) Trigeminal pain pathway: peripheral and central activation as experimental models of migraine. Funct Neurol 16:77-81.

Buzzi MG, Bonamini M, Moskowitz MA (1995) Neurogenic model of migraine. Cephalalgia 15:277-280.

Cau E, Cararosa S, Guillemot F (2002) Mash1 and Ngn1 control distinct steps of determination and differentiation in the olfactory sensory neuron lineage. Development 129:1871-1880.

Corneliussen B, Holm M, Waltersson Y, Onions J, Hallberg B, Thornell A, Grundstrom T (1994) Calcium/calmodulin inhibition of basic-helixloop-helix transcription factor domains. Nature 368:760-764.

Davis KD, Dostrovsky JO (1988) Cerebrovascular application of bradykinin excites central sensory neurons. Brain Res 446:401-406.

de Bustros A, Baylin SB, Levine MA, Nelkin BD (1986) Cyclic AMP and phorbol esters separately induce growth inhibition, calcitonin secretion, and calcitonin gene transcription in cultured human medullary thyroid carcinoma. J Biol Chem 261:8036-8041.

de Bustros A, Ball DW, Peters R, Compton D, Nelkin BD (1992) Regulation of human calcitonin gene transcription by cyclic AMP. Biochem Biophys Res Commun 198:1157-1164.

Durham PL, Russo AF (1998) Serotonergic repression of mitogen-activated protein kinase control of the calcitonin gene-related peptide enhancer. Mol Endocrinol 12:1002-1009.

Durham PL, Russo AF (1999) Regulation of calcitonin gene-related peptide secretion by a serotonergic antimigraine drug. J Neurosci 19:3423-3429.

Durham PL, Russo AF (2000) Differential regulation of mitogen-activated protein kinase responsive genes by the duration of a calcium signal. Mol Endocrinol 14:1570-1582.

Durham PL, Russo AF (2002) New insights into the molecular actions of serotonergic antimigraine drugs. Pharmacol Ther 94:77-92.
Durham PL, Sharma R, Russo AF (1997) Repression of the calcitonin generelated peptide promoter by $5-\mathrm{HT}_{1}$ receptor activation. J Neurosci 17:9545-9553.

Edvinsson L, Goadsby PJ (1994) Neuropeptides in migraine and cluster headache. Cephalalgia 14:320-327.

Eltorp CT, Jansen-Olesen I, Hansen AJ (2000) Release of calcitonin generelated peptide (CGRP) from guinea pig dura mater in vitro is inhibited by sumatriptan but unaffected by nitric oxide. Cephalalgia 20:838-844.

Fanciullacci M, Alessandri M, Figini M, Geppetti P, Michelacci S (1995) Increase in plasma calcitonin gene-related peptide from the extracerebral circulation during nitroglycerin-induced cluster headache attack. Pain 60:119-123.

Ferrari MD (1998) Migraine. Lancet 351:1043-1051.

Fowler PA, Lacey LF, Thomas V, Keen ON, Tanner RJ, Baber NS (1991) The clinical pharmacology, pharmacokinetics and metabolism of sumatriptan. Eur Neurol 31:291-294.

Freeland K, Liu YZ, Latchman DS (2000) Distinct signaling pathways mediate the cAMP response element (CRE)-dependent activation of the calcitonin gene-related peptide gene promoter by cAMP and nerve growth factor. Biochem J 345:233-238.

Galibert MD, Carreira S, Goding CR (2001) The Usf-1 transcription factor is a novel target for the stress-responsive p38 kinase and mediates UVinduced tyrosinase expression. EMBO J 20:5022-5031.

Goadsby PJ, Edvinsson L (1991) Sumatriptan reverses the changes in calcitonin gene-related peptide seen in the headache phase of migraine. Cephalalgia 11 [Suppl 11]:3-4.

Goadsby PJ, Edvinsson L (1993) The trigeminovascular system and migraine: studies characterizing cerebrovascular and neuropeptide changes seen in humans and cats. Ann Neurol 33:48-56.

Goadsby PJ, Edvinsson L (1994) Human in vivo evidence for trigeminovascular activation in cluster headache: neuropeptide changes and effects of acute attacks therapies. Brain 117:427-434.

Hou M, Kanje M, Longmore J, Tajti J, Uddman R, Edvinsson L (2001) 5-HT(1B) and 5-HT(1D) receptors in the human trigeminal ganglion: co-localization with calcitonin gene-related peptide, substance $\mathrm{P}$ and nitric oxide synthase. Brain Res 909:112-120.

Kai J (1993) Extracranial blood flow, pain and tenderness in migraine: clinical and experimental studies. Acta Neurol Scand 88:1-27.

Lanigan TM, Russo AF (1997) Binding of upstream stimulatory factor and a cell-specific activator to the calcitonin/calcitonin gene-related peptide enhancer. J Biol Chem 272:18316-18324.

Lassen LH, Haderslev PA, Jacobsen VB, Iversen HK, Sperling B, Olesen J (2001) CGRP may play a causative role in migraine. Cephalalgia 22:54-61.

Lindsay RM, Harmar AJ (1989) Nerve growth factor regulates expression of neuropeptide genes in adult sensory neurons. Nature 337:362-364.

Ma Q, Chen Z, del Barco Barrantes I, de la Pompa JL, Anderson DJ (1998) Neurogenin 1 is essential for the determination of neuronal precursors for proximal cranial sensory ganglia. Neuron 20:469-482.

Monia YT, Peleg S, Gagel RF (1995) Cell type-specific regulation of transcription by cyclic adenosine $3^{\prime}, 5^{\prime}$-monophosphate-responsive elements within the calcitonin promoter. Mol Endocrinol 9:784-793.

Moskowitz MA (1993) Neurogenic inflammation in the pathophysiology and treatment of migraine. Neurology 43:S16-S20.

Onions J, Hermann S, Grundstrom T (2000) A novel type of calmodulin interaction in the inhibition of basic helix-loop-helix transcription factors. Biochemistry 39:4366-4374.

Pang L, Sawada T, Decker SJ, Saltiel AR (1995) Inhibition of MAP kinase kinase blocks the differentiation of PC-12 cells induced by nerve growth factor. J Biol Chem 270:13585-13588.

Patel TD, Jackman A, Rice FL, Kucera J, Snider WD (2000) Development of sensory neurons in the absence of NGF/TrkA signaling in vivo. Neuron 25:345-357.

Peleg S, Abruzzese RV, Cote GJ, Gagel RF (1990) Transcription of the human calcitonin gene is mediated by a $\mathrm{C}$ cell-specific enhancer containing E-box-like elements. Mol Endocrinol 4:1750-1757.

Russo AF, Lanigan TM, Sullivan BE (1992) Neuronal properties of a thyroid C-cell line: partial repression by dexamethasone and retinoic acid. Mol Endocrinol 6:207-218.

Russo AF, Clark MS, Durham PL (1996) Thyroid parafollicular cells. An accessible model for the study of serotonergic neurons. Mol Neurobiol 13:257-276. 
Sarchielli P, Alberti A, Floridi A, Gallai V (2001) Levels of nerve growth factor in cerebrospinal fluid of chronic daily headache patients. Neurology 57:132-134.

Shadiack AM, Sun Y, Zigmond RE (2001) Nerve growth factor antiserum induces axotomy-like changes in neuropeptide expression in intact sympathetic and sensory neurons. J Neurosci 21:363-371.

Stewart WF, Schechter A, Rasmussen BK (1994) Migraine heterogeneity: disability, pain intensity, and attack frequency and duration. Neurology 44:S24-S39.

Supowit SC, Zhao H, DiPette DJ (2001) Nerve growth factor enhances calcitonin gene-related peptide expression in the spontaneously hypertensive rat. Hypertension 37:728-732.

Tabuchi A, Sakaya H, Kisukeda T, Fushiki H, Tsuda M (2002) Involvement of an upstream stimulatory factor as well as cAMP-responsive elementbinding protein in the activation of brain-derived neurotrophic factor gene promoter I. J Biol Chem 277:35920-35931.

Thiagalingam A, deBustros A, Borges M, Jasti R, Compton D, Diamond L, Mabry M, Ball DW, Baylin SB, Nelkin BD (1996) RREB-1, a novel zinc finger protein, is involved in the differentiation response to ras in human medullary thyroid carcinomas. Mol Cell Biol 16:5335-5345.

Tverberg LA, Russo AF (1993) Regulation of the calcitonin/calcitonin generelated peptide gene by cell-specific synergy between helix-loop-helix and octamer-binding transcription factors. J Biol Chem 268:15965-15973.

Van Rossum D, Hanisch UK, Quirion R (1997) Neuroanatomical localization, pharmacological characterization and functions of CGRP, related peptides and their receptors. Neurosci Biobehav Rev 21:649-678.

Verge VM, Richardson PM, Wiesenfeld-Hallin Z, Hokfelt T (1995) Differential influence of nerve growth factor on neuropeptide expression in vivo: a novel role in peptide suppression in adult sensory neurons. J Neurosci 15:2081-2096.

Watson A, Ensor E, Symes A, Winter J, Kendall G, Latchman D (1995) A minimal CGRP gene promoter is inducible by nerve growth factor in adult rat dorsal root ganglion neurons but not in PC12 phaeochromocytoma cells. Eur J Neurosci 7:394-400.

Williamson DJ, Hargreaves RJ (2001) Neurogenic inflammation in the context of migraine. Microsc Res Tech 53:167-178. 